\title{
Images Perceived After Chromatic or Achromatic Contrast Sensitivity Losses
}

\author{
M. José Luque*, Pascual Capilla*, M. Dolores de Fez*, and M. Carmen García-Domene ${ }^{\dagger}$
}

Purpose. We simulate how subjects with losses in chromatic and achromatic contrast sensitivity perceive colored images by using the spatiochromatic corresponding pair algorithm.

Methods. This is a generalized version of the algorithm by Capilla et al. (J Opt Soc Am (A) 2004;21:176-186) for simulating color perception of color deviant subjects, which incorporates a simple spatial vision model, consisting of a linear filtering stage, with a band-pass achromatic filter and two low-pass chromatic ones, for the red-green and blue-yellow mechanisms. These filters, except for the global scaling, are the subject's contrast sensitivity functions measured along the cardinal directions of the color space. In its present form, the algorithm would serve to simulate alterations both in the spectral sensitivities and in the contrast sensitivities of the visual mechanisms.

Results. After a preliminary theoretical study on the effect of frequency selective and overall reductions in the contrast sensitivity function of a single mechanism, we present cases of real subjects with glaucoma and diabetes, suffering alterations of different magnitude in the three mechanisms.

Conclusions. The simulations allow us to learn about the different types of distortions that can be experienced by a subject with impaired contrast sensitivities (blur, haloes, color shifts, local or global contrast, brightness and colorfulness reductions, etc.) and highlight the difficulties arising when trying to predict the quality of the final image from the losses in the individual mechanisms.

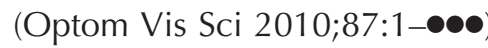

Key Words: chromatic and achromatic CSFs, images simulation, glaucoma, diabetes, corresponding pair algorithm

A great variety of visual pathologies (glaucoma, optic neuritis, etc.) and pathologies not primarily of visual origin but impairing vision at short- or long-term (diabetes, multiple sclerosis, etc.) provoke changes in the relative action spectra of the chromatic and achromatic color mechanisms ${ }^{1-9}$ and, also, selectively reduce chromatic and achromatic contrast sensitivity for certain spatiotemporal frequencies. ${ }^{10-21}$ As a result, the same image may appear very different in various aspects to an observer suffering these impairments and to a normal observer.

The reported functional impairments have been often related to relative losses of ganglion cells in the magnocellular, parvocellular, and koniocellular pathways ${ }^{22-26}$ mediating the achromatic, the red-green, and the blue-yellow mechanisms, respectively. However, alterations in the spectral sensitivity (and, therefore, in the action spectra) of a mechanism may have their origin in the cones.

\section{$* \mathrm{PhD}$}

${ }^{\dagger} \mathrm{OD}$

Departament d'Òptica, Universitat de València, València, Spain (MJL, PC), Departamento de Óptica, Farmacología y Anatomía, Universidad de Alicante, Alicante, Spain (DdF), and Fundación Oftalmológica del Mediterráneo, Alicante, Spain (MCG-D).
For instance, the performance of a red-green mechanism may be abnormal as the result of a change in the total number or in the relative proportion of functional $\mathrm{L}$ and $\mathrm{M}$ cones, which happens in persons with variant cone spectral sensitivities (as in anomalous and dichromatic observers) ${ }^{27}$ or in observers with selective cone dystrophies. ${ }^{28,29}$ It is even less trivial to relate changes in contrast sensitivity with certain anatomical losses, because the contrast sensitivity function (CSF) is actually the envelope of the frequency response curves of cortical cells tuned to different frequency bands and the observed alteration may have retinal or cortical origin. ${ }^{30-35}$ Taking all this into account, an algorithm to simulate image appearance for observers with real visual impairment ought to make use of a vision model allowing the introduction of local or global changes at different visual information processing stages (preretinal, retinal, and cortical).

In this work, we propose a modification of the corresponding pair algorithm ${ }^{36,37}$ working with color and spatial processing models, and which we call spatiochromatic corresponding pair algorithm, to simulate how observers with a variety of vision defects perceive a colored scene. Although a full form of the algorithm is presented, for the examples shown in this article, the algorithm is 
2 Perception After Chromatic or Achromatic Contrast Sensitivity Losses-Luque et al.

implemented on a simplified model for normal and variant vision, consisting essentially of a linear filtering stage, with a band-pass achromatic filter and two low-pass chromatic ones for the redgreen and blue-yellow mechanisms. Although we are assuming here separate spatial and color processing and separate chromatic and achromatic processing, more sophisticated models can be used to include multichannel processing, ${ }^{38-40}$ nonlinear contrast-gain control, ${ }^{41-43}$ scaling of the receptive-field sizes with eccentricity, ${ }^{44}$ and interactions between chromatic and achromatic mechanisms, ${ }^{45,46}$ provided certain mathematical restrictions are fulfilled. ${ }^{36,37}$ However, we suggest that, despite these simplifications, the algorithm in its present form can be used to gain some insight about how observers with vision defects see natural scenes. We analyze the effect of systematic changes in each individual mechanism and present results for two patients with glaucoma (early and advanced) and two patients with diabetes (with and without retinopathy) to illustrate the combined effect of impairments in the three cardinal mechanisms, which is the case of most real observers. Preliminary results of this study were presented at the 2008 Conference on Color in Graphics, Imaging and Vision. ${ }^{47}$

\section{METHODS}

\section{The Spatiochromatic Corresponding Pair Algorithm}

The corresponding pair algorithm has been proposed as a method for simulating images perceived by dichromatic observers of any type, using any color vision model, represented by a mathematical operator $\mathrm{m}$, with normal and dichromatic versions, provided the normal model can be inverted. ${ }^{36,37}$ We have reformulated the algorithm (Eq. 1) to simulate how any observer with variant vision perceives a scene. The appearance a stimulus $T(x, y)$ has for a variant observer can be described if a stimulus $S(x, y)$ is found verifying that a normal observer experiences the same sensation viewing $\mathrm{S}(\mathrm{x}, \mathrm{y})$ as the observer viewing $\mathrm{T}(\mathrm{x}, \mathrm{y})$. If normal and variant versions of the same vision model $\mathrm{m}$ are available, the tristimulus values of $S(x, y)$ are obtained by applying the inverse of the normal model to the perceptual descriptors of $\mathrm{T}(\mathrm{x}, \mathrm{y})$ computed with the variant model, that is:

$$
\mathrm{S}(\mathrm{x}, \mathrm{y})=\mathrm{m}^{-1}\left(\mathrm{~m}\left(\mathrm{~T}(\mathrm{x}, \mathrm{y}), \mathrm{p}_{\mathrm{v}}\right), \mathrm{p}_{\mathrm{n}}\right)
$$

where $T(x, y)$ and $S(x, y)$ are the tristimulus values of the original and simulated stimulus at each spatial position $(\mathrm{x}, \mathrm{y}), \mathrm{m}$ is a mathematical operator comprising all the transformations of the vision model, and $\mathrm{p}_{\mathrm{n}}$ and $\mathrm{p}_{\mathrm{v}}$ are, the set of parameters defining the normal and variant versions of the model, respectively. Note that operator $\mathrm{m}$ is not constrained to a single processing stage but may comprise preretinal, retinal, and cortical stages and that $\mathrm{p}$ is the set of parameters controlling all the properties of all the stages incorporated by the model. The computational cost of Eq. 1 may be reduced if the normal and the variant models coincide beyond a given stage, because it would suffice to compute the inverse of the output of the last stage where the models differ.

The vision model m must satisfy, at least, the following conditions: (1) the normal version of the model must be invertible, either analytically or numerically; (2) normal and variant versions must provide the same number of descriptors to avoid an infinite number of solutions in Eq. 1; and (3) the values of the descriptors computed for the variant observer, for each stimulus, must lie within the range of those for the normal observer to avoid having "unreal colors" as solutions. Besides verifying these mathematical requirements, the model ought to be as complete and possible and to allow manipulation of the site where the pathology is supposed to occur. The simulation obtained, of course, depends on the model. The richer the model, the more accurate the prediction made with the algorithm. In fact, testing the validity of the predictions of Eq. 1 would serve as a test of the validity of a given vision model ${ }^{36,37}$ (please, check the Conclusions section for further details).

In this work, we have assumed that in the normal and variant version of model, $\mathrm{m}$ pattern and color processing are separable. Pattern-color separability has been tested for both appearance and sensitivity at least for simple patterns ${ }^{48,49}$ and is assumed for more complex patterns in widely used vision models, such as S-CIELAB. ${ }^{50}$ In this way, the alterations suffered by an observer may be simulated as arising from changes affecting spectral sensitivity of certain color mechanisms or from changes in contrast sensitivity of certain spatial mechanisms (which are also color selective). That is,

$$
\mathrm{S}(\mathrm{x}, \mathrm{y})=\mathrm{m}^{-1}\left(\mathrm{~m}\left(\mathrm{~T}(\mathrm{x}, \mathrm{y}), \mathrm{p}_{\mathrm{vc}}\right), \mathrm{p}_{\mathrm{vs}}\right), \mathrm{p}_{\mathrm{nc}}, \mathrm{p}_{\mathrm{ns}}
$$

where $\mathrm{p}_{\mathrm{nc}}$ and $\mathrm{p}_{\mathrm{vc}}$ are the set of parameters defining the spectral sensitivities of the color mechanisms in the normal and variant versions of the model, respectively, and $\mathrm{p}_{\mathrm{ns}}$ and $\mathrm{p}_{\mathrm{vs}}$, the parameters defining their spatial properties. We call Eq. 2 the spatiochromatic corresponding pair algorithm. Although different forms of the model $\mathrm{m}$ can be postulated, in this article, we will only assume that the model provides the responses of an achromatic $(A(x, y))$, a red-green $(T(x, y))$, and a blue-yellow $(\mathrm{D}(\mathrm{x}, \mathrm{y}))$ mechanism for each point of the image and that spatial processing is performed separately in outputs of these three mechanisms (Fig. 1). In previous F1 articles, we have explored the effect of changes in the spectral sensitivities of the color mechanisms at different stages and with different models, ${ }^{36,37}$ and in this work, we intend to focus on the effects of alterations in their spatial properties.

The color model we have used here is Guth's ATD95. ${ }^{51}$ Briefly, ATD95 is a three-stage model, including a cone stage, computed from the Smith and Pokorny fundamentals, ${ }^{52}$ with a multiplicative adaptation mechanism and a power-type nonlinearity, an intermediate opponent stage with a nonlinearity of the NakaRushton type, ${ }^{53}$ leading to the responses of the achromatic $\left(A_{1}\right)$, red-green $\left(\mathrm{T}_{1}\right)$, and blue-yellow $\left(\mathrm{D}_{1}\right)$ mechanisms, simulating ganglion cell processing, and, finally, a second nonlinear opponent-stage leading to the responses of the final achromatic $\left(A_{2}\right)$ and the final chromatic $\left(\mathrm{T}_{2}\right.$ and $\left.\mathrm{D}_{2}\right)$ mechanisms, from which perceptual color descriptors may be computed. This last stage would reflect globally the operations occurring at cortical level. We have already shown that this color vision model is adequate to predict the visual performance of protanopic, deuteranopic, and tritanopic subjects. ${ }^{36,37}$ Satisfying protanopic and deuteranopic versions of ATD95 can be obtained with the cone-substitution hypothesis (for protanopes, L cones contain the $\mathrm{M}$ pigment, and for deuteranopes, $\mathrm{M}$ cones contain the $\mathrm{L}$ pigment, the other cones being normal) if besides the red-green mechanism, which losses its opponency, is nulled. Nulling the $S$ cones (cone-null hypothesis) and the yellow-blue mechanism, we obtain a good tritanopic model. 


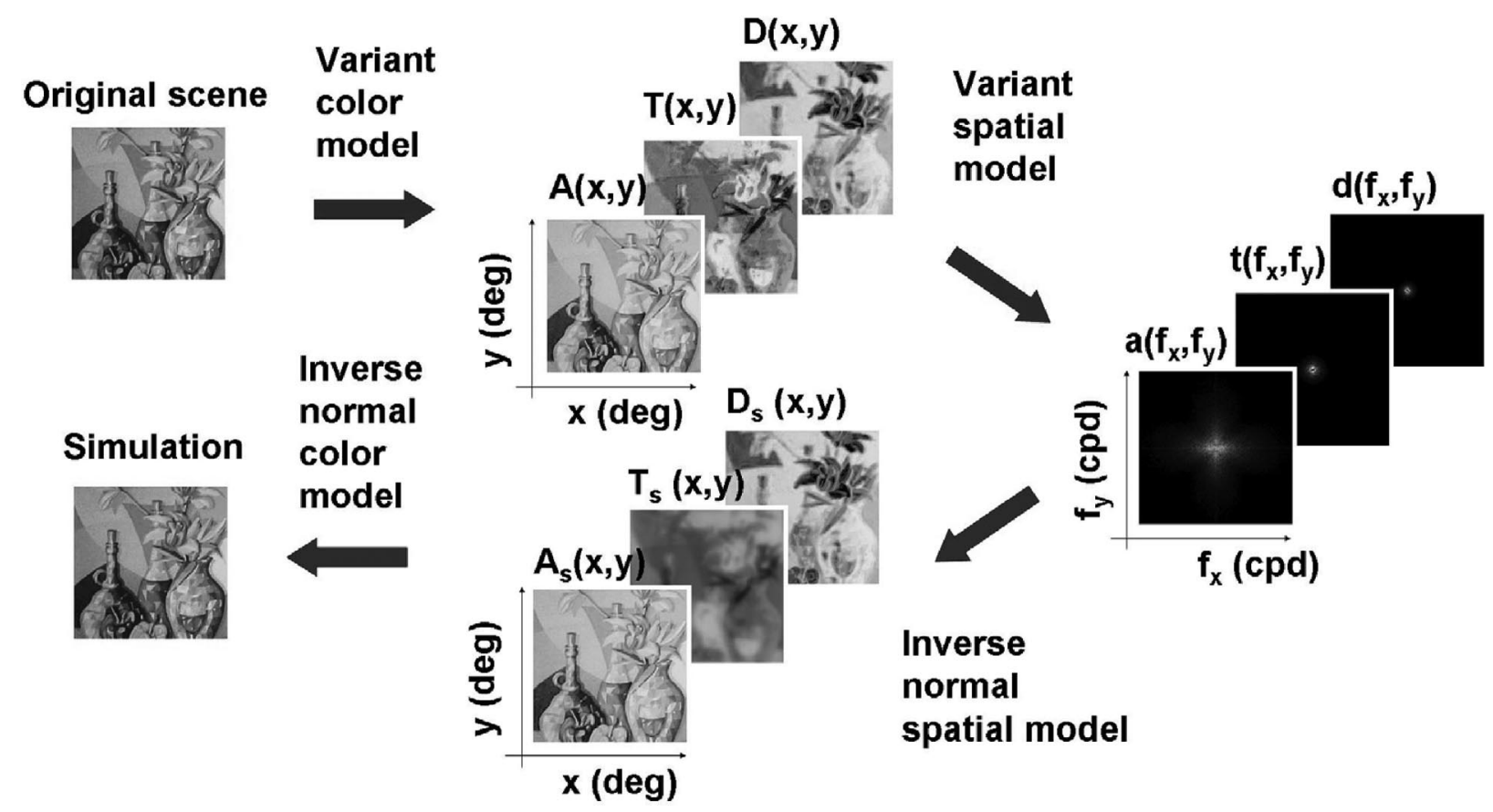

\section{FIGURE 1.}

Schema of the algorithm used. The tristimulus values of the original scene enter the variant version of a color vision model (ATD95) to yield three images, one for the response of each variant color mechanism (achromatic, A, red-green, T, and blue-yellow, D mechanisms). Separate linear variant spatial filters are then applied to each mechanism. The inverse of the normal spatial model yields now the responses of the color mechanisms of a normal observer having the same perception than the variant one. The image that would elicit those responses is computed by inverting the normal color vision model. A color version of this figure is available at www.optvissci.com.

As for the spatial vision model, for simplicity, we have considered a set of three linear filters, one for each of the perceptual mechanisms of the color vision model. These filters are the observer's CSFs, as measured psychophysically with sinusoidal gratings isolating the achromatic, red-green, and blue-yellow mechanisms. ${ }^{54-56}$ The achromatic filter is band-pass peaking around 3 to 4 cycles per degree (cpd) and with a cutoff frequency at $\sim 40 \mathrm{cpd}$, whereas the chromatic red-green and blue-yellow filters are lowpass filters with cutoff frequencies at $\sim 12$ and $10 \mathrm{cpd}$, respectively.

With all this, an image $S(x, y)$ that, for a normal observer, simulates how an image $\mathrm{T}(\mathrm{x}, \mathrm{y})$ appears to a variant observer, is obtained from Eq. 2 as follows:

$$
S_{n, i}(x, y)=\operatorname{FT}^{-1}\left(\operatorname{FT}\left(T_{v, i}(x, y)\right) \frac{\operatorname{CSF}_{v, i}\left(f_{x}, f_{y}\right)}{\operatorname{CSF}_{n, i}\left(f_{x}, f_{y}\right)}\right)
$$

where FT means Fourier transform and subindex i refers to one of the three mechanisms ( $i=A, T$, or D). Subindexes $n$ and $v$ refer to the normal or variant observers. Note that $\mathrm{p}_{\mathrm{ns}}$ and $\mathrm{p}_{\mathrm{vs}}$ are simply $\operatorname{CSF}_{n, i}\left(f_{x}, f_{y}\right)$ and $\operatorname{CSF}_{v, i}\left(f_{x}, f_{y}\right)$, respectively. The values of $\mathrm{p}_{n c}$ and $\mathrm{p}_{\mathrm{vc}}$ in Eq. 2 are not made explicit but are implicit in the transformations that lead to the $\mathrm{A}, \mathrm{T}$, and $\mathrm{D}$ responses. For the simulations that we show here, we will restrict ourselves to the case where $p_{n c}$ and $\mathrm{p}_{\mathrm{vc}}$ are equal, and, thus, all alterations are concentrated in the spatial properties of the mechanisms. Eq. 3 is a straightforward extension to three-dimensional color space of the formula proposed by de Fez et al. ${ }^{57}$ for achromatic images. Note that contrast sensitivity is measured only at a single point, but many pathology involve spatially localized damage. This could not be accounted for by Eq. 3, whose validity is restricted, therefore, to images covering a fairly uniform region of the visual field. However, if the model $\mathrm{m}$ included a mosaic of spatially localized sensors, local damage could be simulated with Eq. 1.

\section{Theoretical Simulations: Data and Procedures}

We have used two different images, one $300 \times 450$ and the other $500 \times 332$ pixels in size, to be viewed at the distance under which the largest side of the image subtends $6^{\circ}$ for the theoretical simulation and $10^{\circ}$ for the cases of real observers. The fast FT of each image was computed, using Matlab, for frequencies in the $\left[-f_{s, i} / 2, f_{s, i} / 2\right]$ interval, where $i$ is the spatial direction ( $x$ or $y$ ) and $\mathrm{f}_{s, \mathrm{i}}$ is the sampling frequency, defined as $\mathrm{N}_{\mathrm{i}} / \alpha_{\mathrm{i}}, \mathrm{N}_{\mathrm{i}}$ being the number of pixels in direction $i$ and $\alpha_{\mathrm{I}}$, the angle subtended by that side of the image.

For the theoretical simulations, we have considered damage in a single mechanism, keeping the other two intact. In the study of the achromatic mechanism, the normal band-pass filter was implemented as a difference of two Gaussians reproducing the sensitivity of a standard observer, ${ }^{58}$ and the alterations we have considered were either global sensitivity reductions or losses at localized frequency bands. Because the achromatic CSF can be understood as the envelope of the responses of cortical cells that are spatially selective and, hence, band pass in the frequency domain (for a review, see for example, Wilson and Wilkinson ${ }^{59}$ ), localized sensitivity losses would simulate the effect of damage in cells tuned to some particular frequency. We assumed that these sensors have Gaussian sensitivities in the frequency domain, peaking at $\mathrm{f}_{0}\left(\mathrm{f}_{0}=\right.$ 1,4 , or $16 \mathrm{cpd}$ ) and with a bandwidth $\sigma$ equal to 1 octave. Loss of 
Perception After Chromatic or Achromatic Contrast Sensitivity Losses-Luque et al.

a particular sensor has been simulated by multiplying the normal CSF for the function $1-\mathrm{e}^{-\left(\left(\log _{2} f-\log _{2} f_{o} / \sigma\right)^{2}\right.}$, where $\mathrm{f}$ is spatial frequency. However, losses in a particular band of sensors may have precortical (for information, in optical neuritis ${ }^{60}$ ) or cortical origin; an even optical factors have been reported to alter contrast sensitivity at the low and medium frequency range. ${ }^{61,62}$ Note that the losses we are simulating causes notches in the CSFs, which may be narrower than those reported in the literature, ${ }^{32,63,64}$ but in this way, we obtain more illustrative results. A global reduction in the CSF occurs when the sensitivities of cells tuned to all frequency bands are simultaneously impaired (in our examples, reduced by a $1 / 3$ global factor). We assume in all our variant observers that the sensitivity for the null spatial frequency is the same as for a normal observer, meaning that the mean luminance of the image is not altered. This is a reasonable assumption, because, even if the sensitivity of all the cells were reduced, the zero frequency would remain intact, as there is no sensor particularly tuned to that frequency, and besides, we have not considered another potential source for reductions of mean luminance, such as those of optical origin. We do not mean that the possibility of finding patients with this kind of alteration must be discarded, but simulations describing the perception of such patients would be characterized mainly by global darkening and the rest of the effects being so masked that the result would not be illustrative.

Regarding the chromatic mechanisms, the contrast sensitivity of the normal observer was simulated by a single Gaussian peaking at zero frequency and with different cutoff frequencies for the $\mathrm{T}$ and $\mathrm{D}$ mechanisms - note that the absolute sensitivity is irrelevant for this simulation (see Eq. 3) - and the alterations we have considered include both reductions in the amplitude - that is, reductions of global sensitivity by a constant factor, $\mathrm{k}_{\mathrm{g}}$ - and in the cutoff frequency — reductions of bandwidth by a constant factor $\mathrm{k}_{\mathrm{bw}}$. In this case, the global amplitude reductions do affect the zero frequency. Similar to the achromatic CSF, the chromatic CSF can be understood as the envelope of the responses of band-pass cells tuned to different frequencies, because most cells in V1 receiving inputs of the opposite sign from $\mathrm{L}$ and $\mathrm{M}$ cones have double-opponent receptive fields with imbalanced cone inputs, and therefore, their responses are bandpass both for chromatic and achromatic patterns. ${ }^{65,66}$ These cells are sometimes named color-luminance cells. The low-pass shape of the psychophysical chromatic CSF would be the result of the relative weights of the different sensors contributing to the global response of the visual system. However, some attenuation should appear when the spatial frequency approaches zero, because all these cells, even those tuned to the lowest possible frequency, are band pass. Of course, the psychophysical chromatic CSF might be intrinsically band pass but the low frequency fall off might occur at frequencies far below those used in this kind of measurements and would, therefore, be overlooked. Alternatively, however, the chromatic CSF might be mediated by the responses of a particular class of cortical cells, characterized for being basically blind for achromatic patterns and low pass for chromatic patterns. These cells, named sometimes color cells, may represent a limit of the color-luminance cell population (double-opponent cells), when cone unbalance is minimum (so, they tend to be blind to achromatic patterns) and the contribution of the surround is irrelevant (so, they tend to be low pass for chromatic patterns). ${ }^{65,66}$ The characteristics of this set of cells are similar to those of the cell group found inside the cytochrome-oxidase blobs. ${ }^{67-69}$ When simulating global sensitivity losses in the chromatic mechanisms, we have assumed the second of these two hypotheses.

\section{Simulations with Real Patients: Data and Procedures}

For the simulations with data from real observers, we measured the CSFs in a dark room, with stimuli generated on a 22-in Mitsubishi Diamond Pro 2070 SB CRT monitor at $160 \mathrm{~Hz}$ frame rate, AQ:4 driven by a ViSaGe video controller of 14 bits provided by Cambridge Research Systems. The CRT was colorimetrically characterized and gamma corrected. Stimuli had sinusoidal profiles along one of the three cardinal directions and were presented in a $5 \times 5^{\circ}$ square window, centered in fovea. To avoid temporal transients, Gaussian temporal smoothing at stimulus on- and off-set was introduced. The mean luminance was $40 \mathrm{~cd} / \mathrm{m}^{2}$, and the observer adapted for $3 \mathrm{~min}$ to this luminance before starting measurements. Threshold contrast was determined by an interleaved staircase procedure, with all frequencies appearing in a random sequence. The staircase began for each frequency at the maximum amplitude achievable by the CRT for the selected mechanism. After each trial for a given frequency, amplitude was decreased by a factor ${ }^{2^{k}} \sqrt{2}$ if the stimulus had been detected or increased by the same factor, otherwise $\mathrm{k}$ being the number of reversals in the staircase for that frequency up to that particular trial. Criteria for exiting the staircase were either totalling 4 reversals or 20 trials, and threshold was defined as the amplitude value of the last presentation with positive detection or, if no stimulus had been detected, as the maximum amplitude value along the corresponding direction of color space. The unidimensional normal CSF for each mechanism, CS$F_{m}(f x, 0)$, was obtained as the average of six normal eyes in the 25 to 35 years of age range, whereas for the variant observers, we used the AQ:5 CSFs of two diabetic eyes (D1, with diagnosed retinopathy, and D2) and two glaucomatous eyes (G1 and G2; Table 1 and Fig. 2). T1,F2 In all cases, the necessary bidimensional CSFs were derived from the unidimensional data, admitting rotational symmetry (the oblique effect was, therefore, not considered), and the resulting functions were interpolated and extrapolated to cover the same frequency domain as the FT of the image. None of these particular patients had localized scotomata in the foveal region that would make Eq. 3 invalid. Informed consent was obtained from all participants after the nature and procedures of the study were explained. The study adheres to the tenets of the Declaration of Helsinki for Research Involving Human Subjects.

\section{RESULTS}

\section{Theoretical Simulations}

Although many readers will be familiar with the effects on achromatic images of impairments in the achromatic CSF (blur, haloes, local, and global contrast losses), we show how these changes affect a colored image. In Figure 3b, c, it can be seen that decre- F3 ments in global sensitivity of the achromatic mechanism, with intact chromatic mechanisms, lead, as the gravity of the loss increases, to quasi-isoluminant images, where image segmentation and pattern recognition tasks become increasingly difficult, be- 
TABLE 1.

Characteristics of the patients used in the study.

\begin{tabular}{|c|c|c|c|c|c|c|c|c|c|c|c|c|}
\hline Patient & $\begin{array}{l}\text { Age } \\
(y r)\end{array}$ & Sph (D) & Cyl (D) & Axis $\left(^{\circ}\right)$ & Ad (D) & VA & IOP & $\mathrm{MD}(\mathrm{dB})$ & $\mathrm{MS}(\mathrm{dB})$ & $\operatorname{LV}(\mathrm{dB})$ & Diagnosis & Complications \\
\hline G1 & 67 & -2.5 & -0.50 & 100 & 2.5 & $6 / 6$ & 20 & 0.775 & 16.62 & 14.55 & $\begin{array}{l}\text { Advanced } \\
\text { glaucoma }\end{array}$ & - \\
\hline G2 & 63 & +0.75 & -2.00 & 70 & 2.5 & $6 / 6$ & 17 & -1.89 & 19.74 & 4.142 & $\begin{array}{l}\text { Incipient } \\
\text { glaucoma }\end{array}$ & $\begin{array}{c}\text { Slight papillary } \\
\text { excavation }\end{array}$ \\
\hline D1 & 48 & -2.00 & - & 一 & 1.75 & $6 / 6$ & 20 & 3.429 & 16.06 & 7.626 & $\begin{array}{l}\text { Type II diabetes, } \\
\text { DR }\end{array}$ & $\begin{array}{r}\text { Macular } \\
\text { edema }\end{array}$ \\
\hline D2 & 65 & - & -0.50 & 80 & 1 & $6 / 6$ & 16 & 2.317 & 15.30 & 10.51 & Type I diabetes & Drusen \\
\hline
\end{tabular}

All patients were men, with normal color vision (as determined by the D15 test) and normal central fields (up to $10^{\circ}$ ), as determined with a Topcon SPB perimeter (MD, MS, and LV values were obtained with that perimeter).

Ad, near correction; VA, visual acuity; MS, mean sensitivity; MD, mean defect; LV, loss variance; DR, diabetic retinopathy.
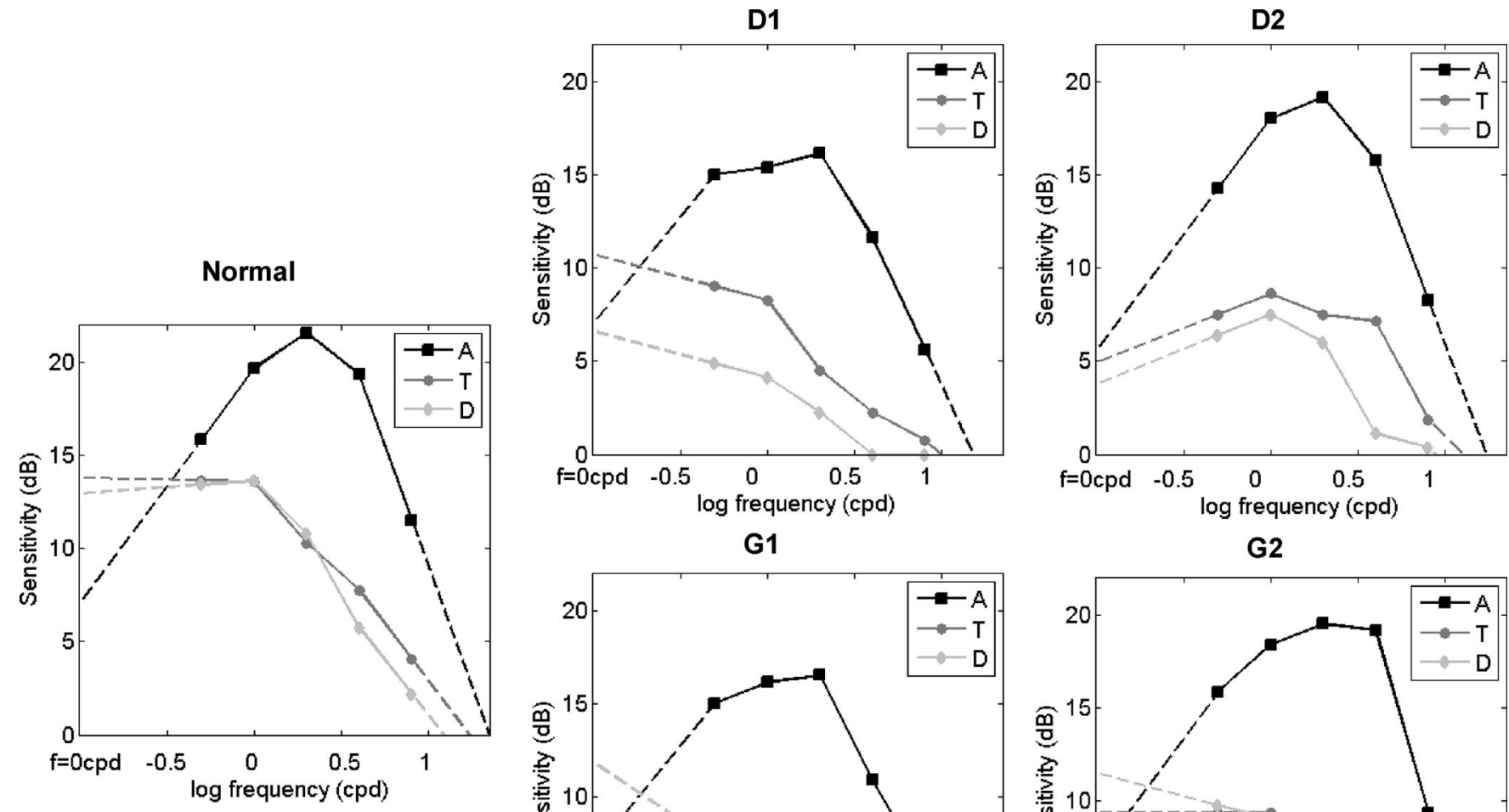

G1

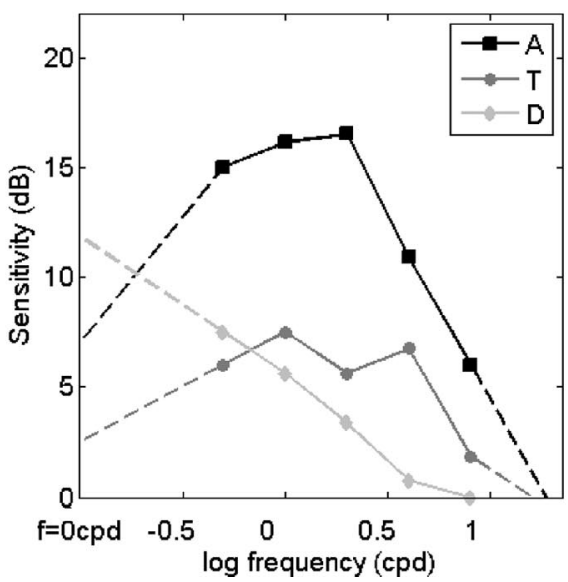

G2

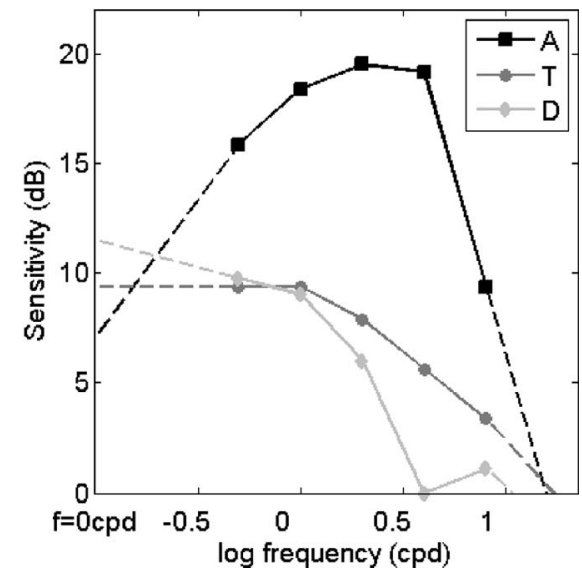

\section{FIGURE 2.}

CSFs for sinusoidal stimuli modulated along the three cardinal directions, obtained for real observers. D1 and D2 are the two diabetic patients and G1 and G2 the two glaucomatous patients. Contrast sensitivity in $\mathrm{dB}$ is computed as $10 * \log _{10}\left(\frac{\text { maximum amplitude }}{\text { threshold amplitude }}\right)$.

cause in the most extreme cases, these tasks would depend only on the comparatively poor spatial information provided by the chromatic mechanisms. In Figure 3d-f, we show the effects of losses in different frequency ranges, which, taking into account the bandpass characteristics of the striate cortex cells-mediating pattern detection, may be thought to correspond with the loss of a set of specific frequency-selective sensors. As it can be seen, losses in the high frequency range result in defocus (Fig. 3f), whereas losses in the low (Fig. 3d) and medium (Fig. 3e) frequency range create dark and bright bands around luminance contours in the image (haloes for short), respectively. Figure $3 \mathrm{~g}-1$ show, as an example, how an observer with normal color vision would perceive a given image, when either the red-green (Fig. 3g-i) or the blue-yellow (Fig. 3j-1) mechanism presents alterations in the corresponding CSFs. For 
6 Perception After Chromatic or Achromatic Contrast Sensitivity Losses-Luque et al.

a

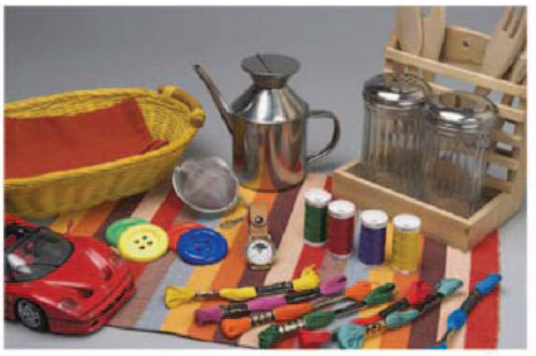

d

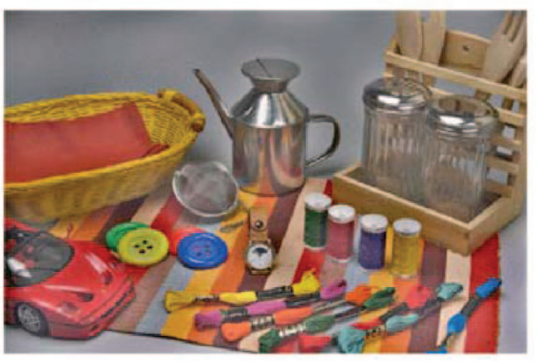

g
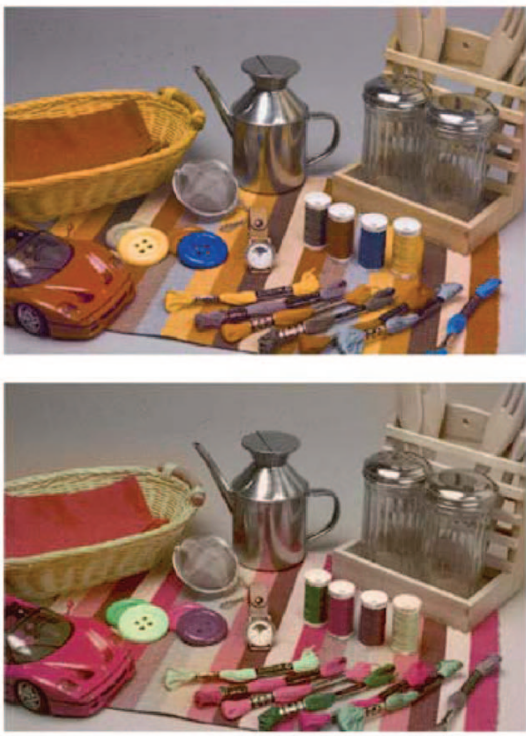

b

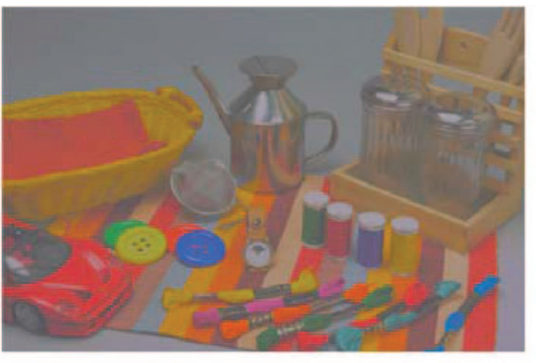

e

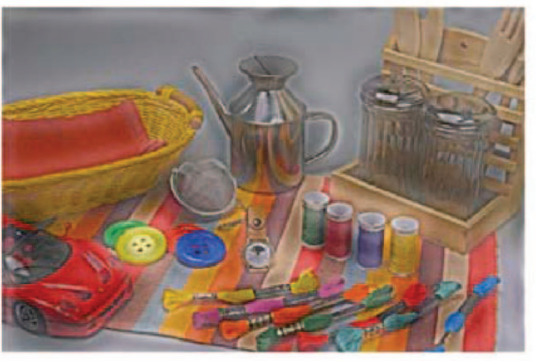

$\mathrm{h}$

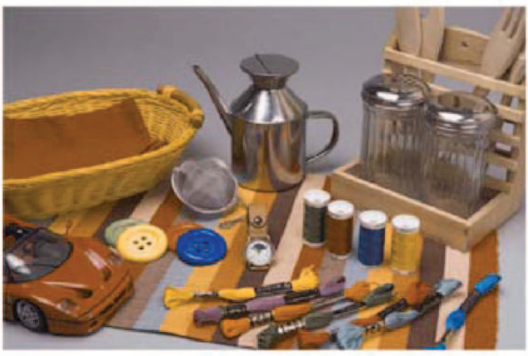

k

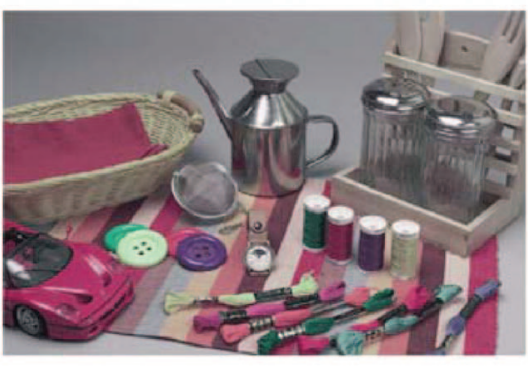

C

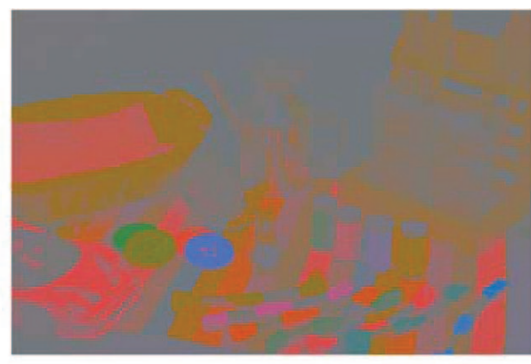

f

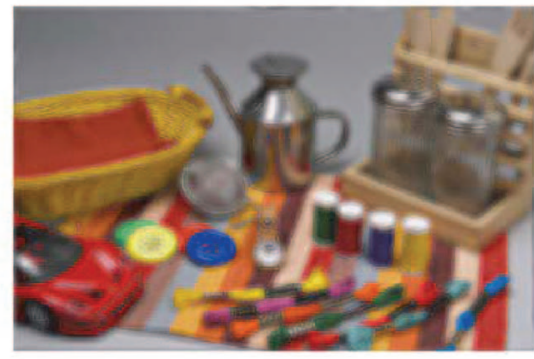

i
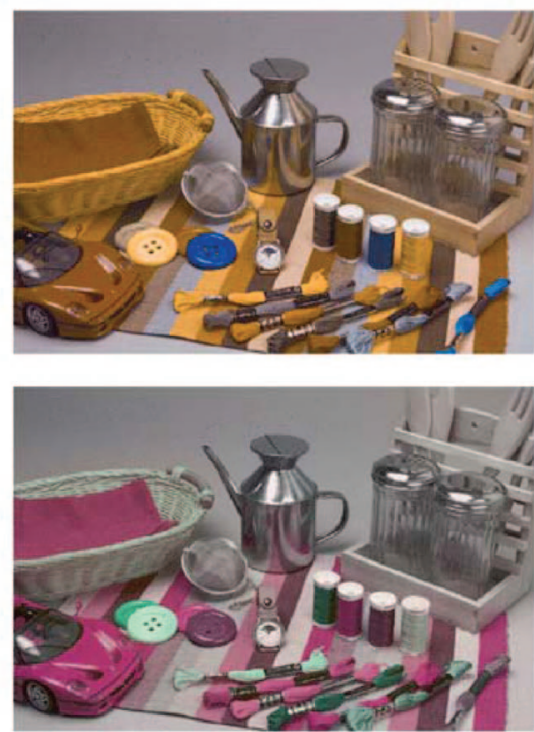

\section{FIGURE 3.}

Effects of different alterations in the achromatic CSF. (a) Original image, (b) moderate global sensitivity reduction, (c) total sensitivity loss of the achromatic mechanism, (d) total loss of the "sensor" tuned at $1 \mathrm{cpd}$, (e) total loss of the sensor tuned at $4 \mathrm{cpd}$, and (f) total loss of the sensor tuned at $16 \mathrm{cpd}$. Combined effects of losses in global sensitivity (constant factor $\mathrm{k}_{\mathrm{g}}$ ) and cutoff frequency (constant factor $\mathrm{k}_{\mathrm{bw}}$ ) in the CSF for the isolated red-green (g-i) and blue-yellow mechanisms (j-l): $(\mathrm{g}-\mathrm{j}) \mathrm{k}_{\mathrm{g}}=1 / 3, \mathrm{k}_{\mathrm{bw}}=1 ;(\mathrm{h}-\mathrm{k}) \mathrm{k}_{\mathrm{g}}=1, \mathrm{k}_{\mathrm{bw}}=1 / 3 ;(\mathrm{i}-\mathrm{l}) \mathrm{k}_{\mathrm{g}}=1 / 3, \mathrm{k}_{\mathrm{bw}}=1 / 3$. Original image reproduced with permission from Hugo Rodriguez (http://www.hugorodriguez.com).

damage in the red-green mechanism (Fig. $3 \mathrm{~g}-\mathrm{i}$ ), as the gravity of the defect increases, oranges and reds become progressively greyer or yellowish, greens become bluish, yellowish, or greyish, and purples become either bluish or greyish. Therefore, the image palette becomes restricted to yellows, blues, and greys. The particular yellow and blue to which the palette converges depend on the color vision model used. Similarly, for damage in the blue-yellow mechanism (Fig. 3j-l), oranges and purples become reddish or greyish, whereas yellows and blues become greenish, reddish, or greyish, and therefore the palette becomes restricted to greens, reds, and greys. Again, the color vision model used determines the chromaticity of the particular green and red to which the palette converges. In Guth's model, these are a bluish-green and a purple. Note that images do not appear defocused, because the achromatic mechanism is intact in these examples.
The presence of alterations in the spatial CSF of one of the chromatic mechanisms makes the perception of the observer similar to that of red-green or blue-yellow defectives (see Fig. 4). F4 Protanopes (Fig. 4d) have been simulated by assuming that the cones that should contain $\mathrm{M}$-wavelength-sensitive photopigment contain L-wavelength-sensitive photopigment (cone substitution hypothesis) and that the red-green mechanism is lost. Analogously, deuteranopes (Fig. 4e) are supposed to have L-wavelength-sensitive photopigment instead of $\mathrm{M}$ and to be also deprived of a red-green mechanism. Tritanopes (Fig. 4f) are assumed to have no Swavelength-sensitive photopigment (cone loss hypothesis) and to lack the blue-yellow mechanism. Note the reduction in color palette to only two hues both in the images corresponding to alterations in spectral sensitivity (in agreement with the literature $^{36,37,70,71}$ ) and in contrast sensitivity in the same color mech- 
a

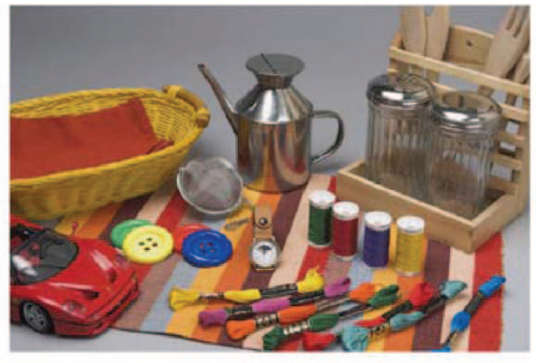

d

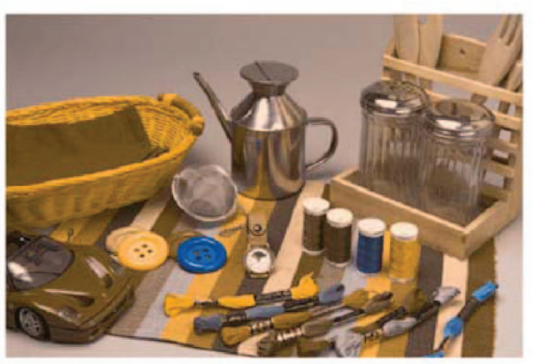

b
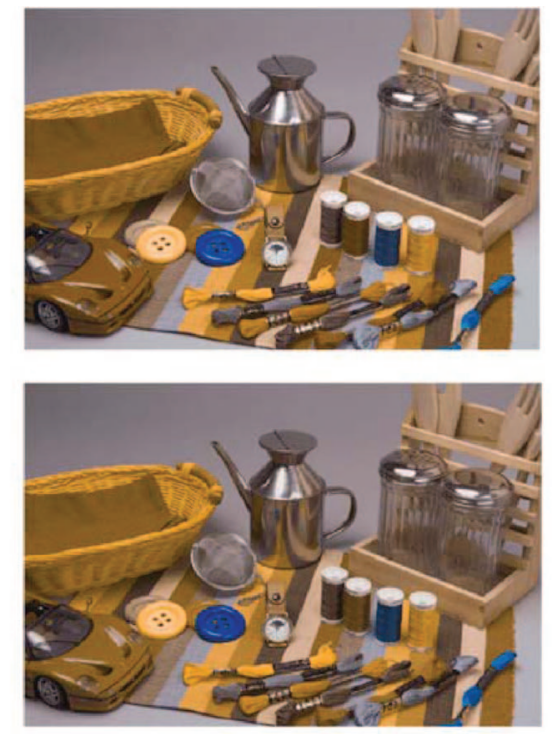

C

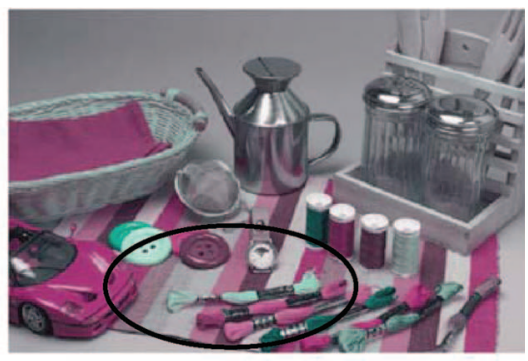

f

\section{FIGURE 4.}

Simulations comparing the effect of alterations in the spectral and in the spatial properties of the color mechanisms. (a) Original image, (b) red-green mechanism with null contrast sensitivity but normal action spectra, (c) blue-green mechanism with null contrast sensitivity but normal action spectra, (d) red-green mechanism with normal contrast sensitivity but the action spectra of a protanope or (e) of a deuteranope, and (f) blue-yellow mechanism with normal contrast sensitivity but the action spectra of a tritanope. The region we have encircled in the image is characterized by the fact that some corresponding pixels have different hues.

b D1

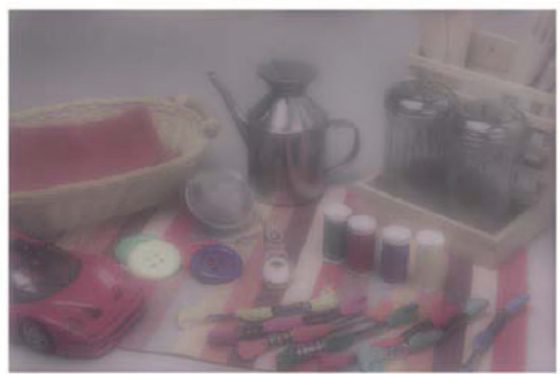

d D2

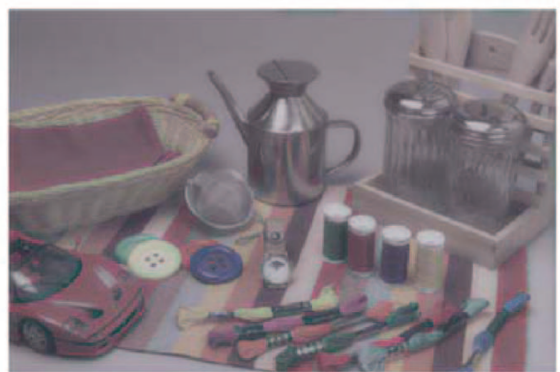

c $\mathbf{G 1}$

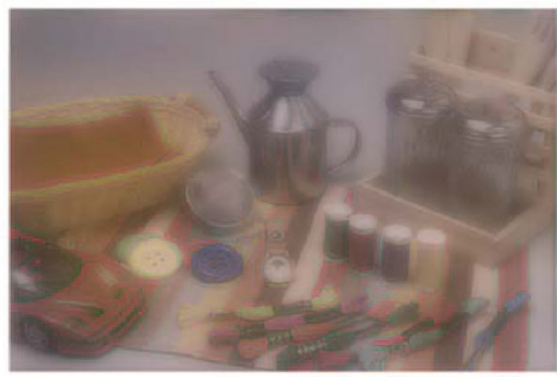

e G2

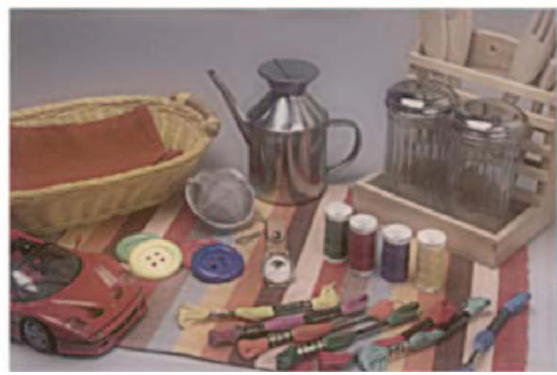

\section{FIGURE 5.}

Simulations of the appearance of the same original image (4a) for different real pathological observers.

anisms. These hues are a particular blue and a particular yellow for loss of the red-green mechanism and a particular red and a particular green for loss of the blue-yellow mechanism. However, if we compare both images by pixel to pixel, we may find differences in brightness, colorfulness, and even hue, because parameters $\mathrm{p}_{\mathrm{nc}}$ and $\mathrm{p}_{\mathrm{vc}}$ in Eq. 2 are identical when the observer has color mechanisms with normal action spectra but not in the case of dichromats. For instance, in Figure 4d, e, it can be seen that protanopes see reds darker than deuteranopes (check the color of the toy car or of the napkin inside the basket), whereas the converse holds for certain greens (one of the buttons, for instance). However, an observer deprived of the same opponent mechanism (T) but with normal cone action spectra shows a behavior that is intermediate between the two red-green defectives (Fig. 4b). More drastic color changes, involving both hue and brightness and colorfulness, are apparent in the case of the tritanope (Fig. 4f) and in the observer deprived of the D mechanism but with normal cone action spectra (Fig. 4c): regions that appear greenish for the first observer are purplish for 
8 Perception After Chromatic or Achromatic Contrast Sensitivity Losses-Luque et al.

the second one (check the basket and the skein in the center of the image).

\section{Simulations with Real Patients}

Finally, in Figure 5a-h, we show simulations for two patients with glaucoma and two patients with diabetes, whose CSFs have been measured in our laboratory (Fig. 2). We have assumed that the chromatic and achromatic mechanisms to have intact spectral sensitivities. It must be noted that the simulations describe the effects of all the factors introducing differences between the variant and the normal observers, and this includes the age difference. Two images have been used to illustrate this part of the study: in Figure 5a-e, the same image as in the theoretical study has been used, so that the real changes may be understood in terms of the methodical variations we have previously introduced in the theoretical CSFs. With this image, the losses in spatial detail are more easily seen. On the other hand, in Figure $5 \mathrm{f}-\mathrm{h}$, we show the effect of the same alterations in an image with a richer color palette-where different color gamuts are, most conveniently, compartmented in different regions of the image and where the effects on color gamut are more strongly manifest than in the first image.

Both images that simulate the perceptions of the diabetic patient D1 are blurred, and spatial details are lost (check, for instance, the mesh of the infuser, the glass pots, the spools of thread, the pattern of the basket, and the inside of the miniature car in Fig. 5b). Images appear with globally reduced colorfulness (yellows and cyans particularly so), and some colors are darkened (compare the blue button and the oil can in Fig. 5a, b, for instance). Different hue shifts are observed: blues and violets becomes purple, oranges become desaturated red, the difference between reddish and yellowish orange is reduced, and the same happens for the difference between green and yellowish-green and yellow. Curiously, however, certain differences are amplified: for instance, the difference between the violet-blue button and the blue right-hand skein in Figure $5 \mathrm{~b}$ has been increased as the combined result of brightness and chromaticity changes.

The basic trends in the diabetic patient D2 (Fig. 5d) are similar to those of D1, but overall image quality is better (less blur, more spatial detail, and higher overall contrast), although chromatic haloes appear in the frontiers between areas of very different brightness (check, for instance, the black shadows around the red car, which appear with a greenish tinge). However, colorfulness is in general lower, because the contrast sensitivity of this observer at the lowest frequency, both for $\mathrm{T}$ and $\mathrm{D}$, is below that of $\mathrm{D} 1$, although in the rest of the frequency range, his contrast sensitivity is higher. Discrimination among greens, yellowish-green, and yellow is better than those of D1, and the same happens with discrimination between reddish and yellowish oranges.

For the glaucomatous patient G1 (Fig. 5c), the image shows global blurring and chromatic haloes around objects placed against a background of very different brightness. The color palette is of lower colorfulness and dark. Again, different hue shifts are produced: reds, oranges, yellows, and greens become orange, blues become slightly more violet, and violets turn to purple.

The general trends in the glaucomatous patient G2 (Fig. 5e) are similar to those in G1, but G2 exhibits better achromatic contrast discrimination of low frequency details (check, for instance, the threads in the spools, the pattern of the basket, and the glass pots).

\section{CONCLUSIONS}

Even when one has developed some intuition about how changes in the CSF of a single mechanism affect the simulated image, foreseeing the result in image quality of the summed up effects of changes along the three cardinal directions is still difficult. The simulations we propose are useful to gain some insight about the different types of distortions that can be experienced by the observers suffering these alterations. These distortions include blur, chromatic or achromatic haloes, local hue shifts and global and local reductions in contrast, brightness or colorfulness. In addition, simulations may give information about how well a particular observer can perform in a given task in comparison with another observer. For instance, if we asked the observers to discriminate spatial detail in areas of almost uniform chromaticity and to make color based segmentation, patient D2 would perform better than D1 and patient G2 better than G1.

The procedure we have followed to compute the simulated images - the corresponding pair algorithm-can be implemented with any vision model $\mathrm{m}$. The choice of the model, of course, determines the final result. The richer the model, the more accurate the prediction made with the algorithm can be expected to be, and in fact, checking the accuracy of the predictions could be a test of the validity of the model. Testing may be theoretical or experimental. In the first case, it might consist, for instance, in predicting certain known facts of the perception of variant observers, such as the colors that are perceived as equal by normal and dichromatic observers. ${ }^{36,37}$ In the second case, the experiment should be conducted as follows: (1) an observer must be found with a normal eye and a variant one, (2) the algorithm is used to compute the image that seen by the observer's normal eye would match the appearance of a given problem image as seen by the observer's variant eye, and (3) the observer would view each image with the appropriate eye and assess the goodness of the match between both images. The experiment would serve, not to test the algorithm, but as a test of the normal and variant versions of the model used. However, unless the model is completely inaccurate, the algorithm will give significant clues about some of the phenomena experienced by an observer, although only within certain limits set by the region of applicability of the model. For instance, with the particular model we have used, we cannot simulate the effect of local scotomata in the visual field, but that could be done by using a model that includes frequency-selective spatially localized sensors.

\section{ACKNOWLEDGMENTS}

We thank Alberto López, DOO, from the Optometric Clinic (University of Alicante), for his collaboration and, also, the voluntary patients who took part in the experiment.

Received May 19, 2009; accepted December 15, 2009. 


\section{REFERENCES}

1. Sperling HG, Piantanida TP, Garrett DS. An atypical color deficiency with extreme loss of sensitivity in the yellow region of the spectrum. Mod Probl Ophthalmol 1976;17:338-44.

2. King-Smith PE, Rosten JG, Alvarez SL, Bhargava SK. Human vision without tonic ganglion cells? In: Verriest G, ed. Colour Vision Deficiencies. V. Bristol, UK: Hilger; 1980:99-105.

3. Adams AJ. Chromatic and luminosity processing in retinal disease. Am J Optom Physiol Opt 1982;59:954-60.

4. Adams AJ, Rodic R, Husted R, Stamper R. Spectral sensitivity and color discrimination changes in glaucoma and glaucoma-suspect patients. Invest Ophthalmol Vis Sci 1982;23:516-24.

5. Alvarez SL, King-Smith PE, Bhargava SK. Spectral thresholds in macular degeneration. Br J Ophthalmol 1983;67:508-11.

6. Foster DH, Snelgar RS, Heron JR. Nonselective losses in foveal chromatic and luminance sensitivity in multiple sclerosis. Invest Ophthalmol Vis Sci 1985;26:1431-41.

7. Alvarez SL, King-Smith PE. Mysterious sparing of blue sensitivity on yellow backgrounds in dominant optic atrophy. In: Drum B, Verriest G, eds. Colour Vision Deficiencies IX: Proceedings of the Ninth Symposium on Colour Vision Deficiencies, St. John's College, Annapolis, Maryland, MD, USA, July 1-3, 1987. Boston, MA: Kluwer Academic Publishers; 2009:309-16.

8. Greenstein VC, Hood DC, Ritch R, Steinberger D, Carr RE. S (blue) cone pathway vulnerability in retinitis pigmentosa, diabetes and glaucoma. Invest Ophthalmol Vis Sci 1989;30:1732-7.

9. Sharanjeet K, Dickinson CM, O’Donoghue E, Murray IJ. Spectral sensitivity in patients with dysthyroid eye disease. Ophthalmic Physiol Opt 1997;17:232-8.

10. Mullen KT, Plant GT. Colour and luminance vision in human optic neuritis. Brain 1986;109(Pt 1):1-13.

11. Breton ME, Wilson TW, Wilson R, Spaeth GL, Krupin T. Temporal contrast sensitivity loss in primary open-angle glaucoma and glaucoma suspects. Invest Ophthalmol Vis Sci 1991;32:2931-41.

12. Porciatti V, Sartucci F. Retinal and cortical evoked responses to chromatic contrast stimuli. Specific losses in both eyes of patients with multiple sclerosis and unilateral optic neuritis. Brain 1996;119(Pt 3): 723-40.

13. Buttner T, Kuhn W, Muller T, Heinze T, Puhl C, Przuntek H. Chromatic and achromatic visual evoked potentials in Parkinson's disease. Electroencephalogr Clin Neurophysiol 1996;100:443-7.

14. Porciatti V, Di Bartolo E, Nardi N, Fiorentini A. Responses to chromatic and luminance contrast in glaucoma: a psychophysical and electrophysiological study. Vision Res 1997;37:1975-87.

15. Alvarez SL, Pierce GE, Vingrys AJ, Benes SC, Weber PA, King-Smith PE. Comparison of red-green, blue-yellow and achromatic losses in glaucoma. Vision Res 1997;37:2295-301.

16. Fristrom B. Peripheral and central colour contrast sensitivity in diabetes. Acta Ophthalmol Scand 1998;76:541-5.

17. Sartucci F, Murri L, Orsini C, Porciatti V. Equiluminant red-green and blue-yellow VEPs in multiple sclerosis. J Clin Neurophysiol 2001;18:583-91.

18. Ansari EA, Morgan JE, Snowden RJ. Psychophysical characterisation of early functional loss in glaucoma and ocular hypertension. $\mathrm{Br} \mathrm{J}$ Ophthalmol 2002;86:1131-5.

19. Flanagan P, Markulev C. Spatio-temporal selectivity of loss of colour and luminance contrast sensitivity with multiple sclerosis and optic neuritis. Ophthalmic Physiol Opt 2005;25:57-65.

20. Ventura DF, Quiros P, Carelli V, Salomao SR, Gualtieri M, Oliveira AG, Costa MF, Berezovsky A, Sadun F, de Negri AM, Sadun AA. Chromatic and luminance contrast sensitivities in asymptomatic car- riers from a large Brazilian pedigree of 11778 Leber hereditary optic neuropathy. Invest Ophthalmol Vis Sci 2005;46:4809-14.

21. Sakai S, Hirayama K, Ogura K, Sakai N, Sudoh M, Murata N, Iwasaki S. Visual function of a patient with advanced adrenoleukodystrophy: comparison of luminance and color contrast sensitivities. Brain Dev 2008;30:68-72.

22. Quigley HA, Sanchez RM, Dunkelberger GR, L'Hernault NL, Baginski TA. Chronic glaucoma selectively damages large optic nerve fibers. Invest Ophthalmol Vis Sci 1987;28:913-20.

23. Johnson CA. Selective versus nonselective losses in glaucoma. J Glaucoma 1994;3(suppl 1):S32-44.

24. Anderson RS, O’Brien C. Psychophysical evidence for a selective loss of M ganglion cells in glaucoma. Vision Res 1997;37:1079-83.

25. Beirne RO, Logan JF, Zlatkova MB, Jackson AJ, Rankin SJ, Demirel $S$, Anderson RS. Peripheral resolution for achromatic and SWS gratings in early to moderate glaucoma and the implications for selective ganglion cell density loss. Invest Ophthalmol Vis Sci 2003;44: 4780-6.

26. Harwerth RS, Carter-Dawson L, Smith EL III, Barnes G, Holt WF, Crawford ML. Neural losses correlated with visual losses in clinical perimetry. Invest Ophthalmol Vis Sci 2004;45:3152-60.

27. Schwartz SH. Spectral sensitivity of dichromats: role of postreceptoral processes. Vision Res 1994;34:2983-90.

28. Sadowski B, Kellner U, Zrenner E, Foerster MH. Selective cone dystrophy. Invest Ophthalmol Vis Sci 1992;33(suppl):967.

29. Kellner U, Sadowski B, Zrenner E, Foerster MH. Selective cone dystrophy with protan genotype. Invest Ophthalmol Vis Sci 1995; 36:2381-7.

30. Bodis-Wollner I. Visual acuity and contrast sensitivity in patients with cerebral lesions. Science 1972;178:769-71.

31. Bodis-Wollner I. Vulnerability of spatial frequency channels in cerebral lesions. Nature 1976;261:309-11.

32. Bodis-Wollner I, Diamond SP. The measurement of spatial contrast sensitivity in cases of blurred vision associated with cerebral lesions. Brain 1976;99:695-710.

33. Bulens C, Meerwaldt JD, van der Wildt GJ, Keemink CJ. Spatial contrast sensitivity in unilateral cerebral ischaemic lesions involving the posterior visual pathway. Brain 1989;112(Pt 2):507-20.

34. Burr D, Fiorentini A, Spinelli D, Blundo C, Tanzilli P, Diamond M, di Russo F, Morrone C. Selective loss of sensitivity to colour contrast with no observable cerebral lesions. Invest Ophthalmol Vis Sci 2003; 44:E-Abstract 3193.

35. Zele AJ, Pokorny J, Lee DY, Ireland D. Anisometropic amblyopia: spatial contrast sensitivity deficits in inferred magnocellular and parvocellular vision. Invest Ophthalmol Vis Sci 2007;48:3622-31.

36. Capilla P, Diez-Ajenjo MA, Luque MJ, Malo J. Corresponding-pair procedure: a new approach to simulation of dichromatic color perception. J Opt Soc Am (A) 2004;21:176-86.

37. Capilla P, Luque MJ, Díez-Ajenjo MA. Looking for the dichromatic version of a colour vision model. Opt A Pure Appl Opt 2004;6: 906-19.

38. Watson AB. Visual detection of spatial contrast patterns: evaluation of five simple models. Opt Express 2000;6:12-33.

39. Watson AB, Ahumada AJ Jr. A standard model for foveal detection of spatial contrast. J Vis 2005;5:717-40.

40. Pan F, Swanson WH. A cortical pooling model of spatial summation for perimetric stimuli. J Vis 2006;6:1159-71.

41. Heeger DJ. Normalization of cell responses in cat striate cortex. Vis Neurosci 1992;9:181-97.

42. Watson AB, Solomon JA. Model of visual contrast gain control and pattern masking. J Opt Soc Am (A) 1997;14:2379-91.

43. Xing J, Heeger DJ. Center-surround interactions in foveal and peripheral vision. Vision Res 2000;40:3065-72. 
10 Perception After Chromatic or Achromatic Contrast Sensitivity Losses-Luque et al.

44. Watson AB. Estimation of local spatial scale. J Opt Soc Am (A) 1987;4:1579-82.

45. Switkes E, Bradley A, De Valois KK. Contrast dependence and mechanisms of masking interactions among chromatic and luminance gratings. J Opt Soc Am (A) 1988;5:1149-62.

46. Mullen KT, Losada MA. Evidence for separate pathways for color and luminance detection mechanisms. J Opt Soc Am (A) 1994;11: $3136-51$.

47. García-Domene MC, de Fez MD, Luque MJ, Capilla P Simulating images perceived by subjects with abnormal colour or spatial vision. In: CGIV 2008-Fourth European Conference on Colour in Graphics, Imaging, and MCS/08 Vision 10th International Symposium on Multispectral Colour Science (CD-ROM), Barcelona, Spain, June 9-13, 2008. ISBN; 2008:149-52.

48. Poirson AB, Wandell BA. Appearance of colored patterns: patterncolor separability. J Opt Soc Am (A) 1993;10:2458-70.

49. Poirson AB, Wandell BA. Pattern-color separable pathways predict sensitivity to simple colored patterns. Vision Res 1996;36:515-26.

50. Zhang X, Wandell BA. A spatial extensión of CIELAB for digital color-image reproduction. J Soc Information Display 1997;5:61-73.

51. Guth SL. Further applications of the ATD model for colour vision. Proc SPIE-Int Soc Opt Eng 1995;2414:12-26.

52. Smith VC, Pokorny J. Spectral sensitivity of the foveal cone photopigments between 400 and $500 \mathrm{~nm}$. Vision Res 1975;15:161-71.

53. Naka KI, Rushton WA. S-potentials from colour units in the retina of fish (Cyprinidae). J Physiol 1966;185:536-55.

54. Mullen KT. The contrast sensitivity of human colour vision to redgreen and blue-yellow chromatic gratings. J Physiol 1985;359: 381-400.

55. Valverg A, Fosse P, Gjerde T. Chromatic contrast sensitivity without correction for chromatic aberration. Invest Ophthalmol Vis Sci 1997; 38:S893.

56. Webster MA, De Valois KK, Switkes E. Orientation and spatialfrequency discrimination for luminance and chromatic gratings. J Opt Soc Am (A) 1990;7:1034-49.

57. de Fez MD, Luque MJ, Viqueira V. Enhancement of contrast sensitivity and losses of chromatic discrimination with tinted lenses. Optom Vis Sci 2002;79:590-7.

58. Watson AB, Ramirez C. A standard observer for spatial vision based on ModelFest dataset. Optical Society of America Annual Meeting, Digest of Technical Papers, SuC6.

59. Wilson HR, Wilkinson F. Spatial channels in vision and spatial pool- ing. In: Chalupa LM, Werner JS, eds. The Visual Neurosciences, vol II. Cambridge, MA: MIT Press; 2004:1060-8.

60. Hess RF, Plant GT. The psychophysical loss in optic neuritis: spatial and temporal aspects. In: Hess RF, Plant GT, eds. Optic Neuritis. Cambridge, UK: Cambridge University Press; 1986:109-51.

61. Hess RF, Carney LG. Vision through an abnormal cornea: a pilot study of the relationship between visual loss from corneal distortion, corneal edema, keratoconus, and some allied corneal pathology. Invest Ophthalmol Vis Sci 1979;18:476-83.

62. Bour LJ, Apkarian P. Selective broad-band spatial frequency loss in contrast sensitivity functions. Comparison with a model based on optical transfer functions. Invest Ophthalmol Vis Sci 1996;37: 2475-84.

63. Regan D, Silver R, Murray TJ. Visual acuity and contrast sensitivity in multiple sclerosis - hidden visual loss: an auxiliary diagnostic test. Brain 1977;100:563-79.

64. Regan D, Raymond J, Ginsburg AP, Murray TJ. Contrast sensitivity, visual acuity and the discrimination of Snellen letters in multiple sclerosis. Brain 1981;104:333-50.

65. Johnson EN, Hawken MJ, Shapley R. The spatial transformation of color in the primary visual cortex of the macaque monkey. Nat Neurosci 2001;4:409-16.

66. Johnson EN, Hawken MJ, Shapley R. Cone inputs in macaque primary visual cortex. J Neurophysiol 2004;91:2501-14.

67. Livingstone MS, Hubel DH. Anatomy and physiology of a color system in the primate visual cortex. J Neurosci 1984;4:309-56.

68. Ts'o DY, Gilbert CD. The organization of chromatic and spatial interactions in the primate striate cortex. J Neurosci 1988;8: 1712-27.

69. Landisman CE, Ts'o DY. Color processing in macaque striate cortex: electrophysiological properties. J Neurophysiol 2002;87:3138-51.

70. Brettel H, Vienot F, Mollon JD. Computerized simulation of color appearance for dichromats. J Opt Soc Am (A) 1997;14:2647-55.

71. Vienot F, Brettel H, Ott L, Ben M'Barek A, Mollon JD. What do colour-blind people see? Nature 1995;376:127-8.

M. Dolores de Fez Departamento de Optica Farmacología y Anatomía Universidad de Alicante Carretera de San Vicente del Raspeig s/n Alicante 03690, Spain e-mail:dolores.fez@ua.es 